\title{
Criminologie
}

\section{Littérature carcérale québécoise}

\section{Lise Brunet-Aubry}

Volume 9, numéro 1-2, 1976

L’emprisonnement au Québec

URI : https://id.erudit.org/iderudit/017057ar

DOI : https://doi.org/10.7202/017057ar

Aller au sommaire du numéro

Éditeur(s)

Les Presses de l'Université de Montréal

ISSN

0316-0041 (imprimé)

1492-1367 (numérique)

Découvrir la revue

Citer ce compte rendu

Brunet-Aubry, L. (1976). Compte rendu de [Littérature carcérale québécoise].

Criminologie, 9(1-2), 191-195. https://doi.org/10.7202/017057ar d'utilisation que vous pouvez consulter en ligne.

https://apropos.erudit.org/fr/usagers/politique-dutilisation/ 
Traiter des prisons est certes important, mais laisser la parole aux prisonniers est parfois plus fondamental encore. En effet, ils sont mieux placés que quiconque pour livrer leurs expériences vécues.

Nous avons réuni ici 10 livres écrits par des détenus des prisons et pénitenciers du Québec. La majorité de ces écrits ont pour but d'attirer l'attention du public et du gouvernement sur le sort que l'on réserve aux détenus de nos institutions pénitentiaires. Tous ces récits permettent de découvrir la vie de ces hommes dont l'univers est limité à celui de leur cellule...

BOYER, Raymond (1972) : Barreaux de fer, hommes de chair, Montréal, Éditions du Jour, $136 \mathrm{p}$.

En 1948, Raymond Boyer, accusé de conspiration pour infraction à la loi des secrets officiels, est condamné à une peine pénitentiaire de 2 ans. Ce livre est un compte rendu des 19 mois qu'il a passés au pénitencier de Saint-Vincent-de-Paul de 1948 à 1950. Il y décrit la vie quotidienne carcérale : l'arrivée, le travail dans les ateliers, les repas, les règlements, les sports... Son récit est entrecoupé de courtes biographies de détenus dont il a fait la rencontre à diverses périodes de son incarcération.

En épilogue, l'auteur décrit, à l'intention des lecteurs qui s'intéressent à la pénologie, les aspects les plus marquants du progrès accompli dans le système pénitentiaire canadien depuis 1950 .

CHAMPAGNE, Normand (1975) : 7945 Champagne d'Arabie, Montréal, Éditions Sondec, $191 \mathrm{p}$. 
L'auteur, Normand Champagne, purge une peine d'emprisonnement à perpétuité pour un meurtre commis à Val d'Or en 1971. En 1972, alors incarcéré à l'Institut Archambault (Ste-Anne des Plaines), il s'attaque à son voisin de cellule Léopold Dion connu comme un "tueur d'enfants", et le tue. Un jury le déclare non coupable de ce meurtre pour cause d'aliénation mentale.

En prison, il décide de raconter l'histoire de sa vie. Il retrace d'abord sa jeunesse au sein d'institutions de réforme; il décrit son passage à la vie adulte, ainsi que les faits et gestes des personnes qui l'ont marqué. Il avoue certains crimes : vol par effraction, viol, kidnapping, meurtre, évasions. Il décrit sa propre expérience de la vie en institution (la plupart du temps à St-Vincent-de-Paul, mais aussi à l'Institut Archambault et à l'Institut Pinel). Il dénonce les pratiques utilisées par les gardiens à l'Unité spéciale de correction et à St-Vincent-de-Paul.

Le témoignage de l'auteur a pour but, d'une part, de faire comprendre son comportement qui s'explique en grande partie par l'environnement familial et plus tard institutionnel, et, d'autre part, d'attirer l'attention du public et des autorités gouvernementales sur le régime parfois inapproprié que l'on réserve aux détenus dans les institutions pénitentiaires.

HÉBERT, Jacques (1965) : Trois jours en prison, suivi des souvenirs de prison de Jules Fournier, Montréal, Le Club du Livre du Québec, 128 p.

À la suite d'une accusation de mépris de cour, Jacques Hébert passe trois jours en prison avant d'être libéré sous cautionnement. L'auteur nous raconte les détails de son arrestation, son procès, sa première nuit en cellule au quartier général de la police et les 3 jours qu'il passe à la prison de Québec.

Cette première partie du livre est suivie des «Souvenirs de prison", de Jules Fournier qui, emprisonné pour diffamation en 1909, a publié à sa libération les souvenirs de son séjour de 3 mois en prison. Son récit a été considéré, à une certaine époque comme le plus célèbre des pamphlets politiques qui ait paru au Canada français depuis le début du siècle.

HÉBERT, Jacques (1959) : Scandale à Bordeaux, Montréal, Éditions de l'Homme, $157 \mathrm{p}$. 
Le but que Jacques Hébert se propose dans ce livre est d'alerter l'opinion publique sur les conditions inhumaines de détention existant à la prison de Bordeaux en 1954 et 1957.

Ayant été, à la suite de circonstances exceptionnelles, en possession du dossier d'un détenu, Pierre Dupont, il dénonce le scandale de la détention de cet homme emprisonné sans avoir été condamné et sans même avoir comparu devant un juge, parce que considéré comme inapte psychologiquement à subir un procès.

En racontant la vie de Pierre Dupont, l'auteur analyse «les vices de notre système de protection de l'enfance prétendue illégitimen, souligne la responsabilité de la société à l'égard de la jeunesse délinquante, et décrit la prison de Bordeaux de l'époque en insistant sur son surpeuplement, manque d'hygiène, nourriture inadéquate, etc. Il dénonce, le "trou", les épidémies de suicides, ainsi que son aile psychanalytique où des centaines d'êtres humains sont condamnés à la folie et à la mort lente.

LAPLANTE, Robert (1963) : Ti-Blanc, Mouton noir, Montréal, Éditions de l'Homme, $126 \mathrm{p}$.

Ce volume, publié en 1963, est l'autobiographie d'un homme qui fut condamné à mort en 1953, mais dont la sentence fut commuée en détention à vie. Ti-Blanc (Roger Laplante), matricule 9875, évoque le climat de son enfance malheureuse, puis son adolescence, remplie de solitude, de haine, de misère, de faim et de maladie, et finalement sa première condamnation pour vol. Libéré, il devient soldat, puis déserte et est de nouveau arrêté pour vol. Quelques années plus tard, il est accusé de meurtre et condamné à être pendu.

L'auteur décrit son arrestation, l'attente du procès, le procès, puis la condamnation a la pendaison. Quelques heures avant son exécution, il reçoit un sursis de 3 mois. Trois jours avant l'expiration du sursis, il apprend qu'un second sursis de deux mois lui est accordé. Quinze jours avant la fin du deuxième sursis, il apprend que sa sentence est commuée en emprisonnement à vie au pénitencier de St-Vincent-de-Paul. Quelques pages sont consacrées aux heures que l'auteur a passées dans sa cellule de condamné à mort.

POLLOCK, Donald (1973) : Appelle-moi un bon voleur, StHubert, Québec, Transformation centre d'information, $446 \mathrm{p}$. 
Donald Pollock, un détenu réhabilité, a vécu 18 années de sa vie dans des écoles de réforme, prisons et institutions d'aliénés. Il croit à la réhabilitation et raconte son histoire pour prouver qu'un criminel peut se réhabiliter. L'auteur relate des épisodes de son enfance malheureuse, ses séjours à l'école de réforme et dans diverses prisons des États-Unis, de l'Ontario et du Québec, ainsi que dans les hôpitaux psychiatriques.

JAUVIN, Ben (1973) : De ma cage à ton salon, Montréal, Éditions de l'Heure, $234 \mathrm{p}$.

Condamné à vingt-cinq ans de détention et incarcéré au pénitencier de St-Vincent-de-Paul, l'auteur écrit un journal qui comporte ses réflexions quotidiennes, sur le régime de la prison ainsi que ses souvenirs d'enfance, d'adolescence et de sa vie adulte.

Sorti de prison (1966): Texte intégral d'une interview avec François D. (Matricule 8230), ancien détenu du pénitencier St-Vincent-de-Paul, Montréal, Éditions du Jour.

CORDEAU, Roger (1973) : Prisonnier du bonheur : biographie de Roger Cordeau, Montréal, Presses Sélect, 128 p.

KATZ, Gertrude (1970) : The Time Gatherers, Montréal, Harvest House, 128 p.

En dehors de ces livres, nous reproduisons galement ici, les rapports et études inédites les plus récentes, fait ; au Québec au cours des dernières années.

\section{BIBLIOGRAPHIE}

AUDET, L. et C. SAMSON (1973) : Indications pour une therapie de groupe en milieu pénitentiaire à sécurité maximale: recherche de simulation, Université de Montréal, École de criminologie, 158 p. (Directeur de la recherche : P.M. Lagier).

BELLEROSE, R. et G. FLEURY (1972) : Cours en informatique dans deux pénitenciers du Québec : description des cours et analyse des résultats obtenus, Université de Montréal, École de criminologie, $85 \mathrm{p}$. (Directeur de la recherche : M.R. Lette).

BOUCHARD, C.A. (1966) : les Émeutes dans le cadre de la réforme pénitentiaire, Mémoire de maitrise inédit, Université de Montréal, Département de criminologie. $141 \mathrm{p}$.

CARRIERE, P. et G. JULIEN (1973): Phase pré-expérimentale du Centre de développement correctionnel, Université de Montreal, École de criminologie, 2 vol., (Directeur de la recherche : P.M. Lagier) 
COLLETTE-CARRIERE, R. (1971) : le Tatougge dans la sous-culture carcérale. Mémoire de maîtrise inédit, Université de Montréal, École de criminologie. $137 \mathrm{p}$.

FRECHETTE, M. et F.X. RIBORDY (1970) : Recherche pénitentiaire : rapport final, Université de Montréal. École de criminologie. 68 p. (Directeur de la recherche : D. Szabo)

GAULIN, C. (1973) : les Critères de sélection utilisés au Centre de réception de Saint-Vincent-de-Paul, Mémoire de maîtrise inédit, Université de Montréal, École de criminologie, $138 \mathrm{p}$.

GILBERT, M.L. (1974) : Projet de visite communautaire à Archambault, Mémoire de maîtrise inédit. Université de Montréal. École de criminologie. $107 \mathrm{p}$.

HERNANDEZ, N. (1970) : la Femme incarcérée à Montréal : typologie psycho-sociale, Université de Montréal. Écołe de criminologie. 91 p.

LANDREVILLE, P., C. MENGHILE et P. PÉPIN (1974): Description de la population de l'établissement de détention de Montréal, Université de Montréal, Ecole de criminologie, $53 \mathrm{p}$.

LEMIRE, G. (1966) : la Punition en milieu carcéral, Mémoire de maîtrise inédit, Université de Montréal. Département de criminologie, 104 p.

LETTE, M.-R. (1970) : Psychopathologie carcérale, comportements spontanés el hiérarchie sociale. Première partie : les deux extrémités de l'échelle sociale, Université de Montréal, École de criminologie, 158 p. (Directeur de la recherche : H.F. Ellenberger).

LIGONDE, P. (1970) : le Syndrome carcéral, Thèse de doctorat inédite, Université de Montréal, École de criminologie, $296 \mathrm{p}$.

MARTIN, R. (1968) : les Types d'adaptation en milieu carcéral, Mémoire de maîtrise inédit, Université de Montréal, Département de criminologie, $122 \mathrm{p}$.

PARADIS, G. (1974) : Carcérologie québécoise : le cas de Bordeaux, Mémoire de maîtrise inédit, Université de Montréal, École de criminologie, 325 p.

PAYETTE, A. (1971) : le «Gardien» dans les institutions pénales au Québec, Mémoire de maîtrise inédit. Université de Montréal, École de criminologie, $98 \mathrm{p}$.

ROY, M. (1968) : Évaluation et analyse d'un traitement de groupe en milieu carcéral, Mémoire de maitrise inédit, Université de Montréal, Département de criminologie, $100 \mathrm{p}$.

VILLENEUVE, G. (1973) : le Processus décisionnel des préposés au classement des pénitenciers, Mémoire de maîtrise inédit. Université de Montréal, École de criminologie, $181 \mathrm{p}$.

VUILLET-ALHERITIERE, F. (1974) : Quelques aspects institutionnels de la discipline au Centre Fédéral de Formation, Unversité de Montréal, École de criminologie, $163 \mathrm{p}$.

Voir aussi: l'article de Marc Leblanc: «Inventaire de la recherche criminologique au Québec : 1949-1969n, Acta Criminologica, vol. III, 1970, p. 171-207. 